\title{
Complexity and performance comparison of Filter Bank Multicarrier and OFDM in uplink of multicarrier multiple access networks
}

Citation for published version (APA):

Saeedi Sourck, H., Wu, Y., Bergmans, J. W. M., Sadri, S., \& Farhang-Boroujeny, B. (2011). Complexity and performance comparison of Filter Bank Multicarrier and OFDM in uplink of multicarrier multiple access networks. IEEE Transactions on Signal Processing, 59(4), 1907-1912. https://doi.org/10.1109/TSP.2010.2104148

DOI:

10.1109/TSP.2010.2104148

Document status and date:

Published: 01/01/2011

Document Version:

Publisher's PDF, also known as Version of Record (includes final page, issue and volume numbers)

Please check the document version of this publication:

- A submitted manuscript is the version of the article upon submission and before peer-review. There can be important differences between the submitted version and the official published version of record. People interested in the research are advised to contact the author for the final version of the publication, or visit the $\mathrm{DOI}$ to the publisher's website.

- The final author version and the galley proof are versions of the publication after peer review.

- The final published version features the final layout of the paper including the volume, issue and page numbers.

Link to publication

\footnotetext{
General rights

- You may freely distribute the URL identifying the publication in the public portal. follow below link for the End User Agreement:

www.tue.nl/taverne

Take down policy

If you believe that this document breaches copyright please contact us at:

openaccess@tue.nl

providing details and we will investigate your claim.
}

Copyright and moral rights for the publications made accessible in the public portal are retained by the authors and/or other copyright owners and it is a condition of accessing publications that users recognise and abide by the legal requirements associated with these rights.

- Users may download and print one copy of any publication from the public portal for the purpose of private study or research.

- You may not further distribute the material or use it for any profit-making activity or commercial gain

If the publication is distributed under the terms of Article 25fa of the Dutch Copyright Act, indicated by the "Taverne" license above, please 


\section{Complexity and Performance Comparison of Filter Bank Multicarrier and OFDM in Uplink of Multicarrier Multiple Access Networks}

\author{
Hamid Saeedi-Sourck, Yan Wu, Jan W. M. Bergmans, \\ Saeed Sadri, and Behrouz Farhang-Boroujeny
}

\begin{abstract}
We compare filter bank multicarrier (FBMC) and orthogonal frequency-division multiplexing (OFDM) in the uplink of a multiple access network. Our study reveals that the high sensitivity of OFDM to carrier frequency offset (CFO) among different users and the need for interference cancellation methods to reduce this sensitivity leads to very complex and yet not very high performance systems. In FBMC-based networks, on the other hand, near-perfect performance is achieved without any need for interference cancellation, thanks to the excellent frequency localized filters used in the realization of FBMC systems.
\end{abstract}

Index Terms-Carrier frequency offset (CFO), computational complexity, filter bank multicarrier (FBMC), offset quadrature amplitude modulation (OQAM), orthogonal frequency division multiple access (OFDMA)

\section{INTRODUCTION}

Multicarrier modulation has recently been recognized as an efficient technique for realization of broadband communication systems. Moreover, adoption of multicarrier technologies for multiuser communications has recently been seriously considered by the industry, as exemplified by activities in the third-generation partnership project (3GPP) long-term evolution (LTE) radio standard working group, where orthogonal frequency-division multiple access (OFDMA) has been suggested and studied extensively [1]. In the uplink of a multiuser network, OFDMA faces a major problem: high sensitivity to carrier frequency offsets (CFO) among different users [2]. This may lead to significant multiple-access interference (MAI) among users which should be taken care of by using computationally expensive signal processing compensation techniques [3].

The choice of a CFO compensation method in a base station (BS) is closely related to the adopted subcarrier allocation scheme [3]. Block and block-interleaved allocation schemes have been suggested [1], [4], [5]. However, the current trend in the industry, as reflected in the 3GPP LTE documents, is more towards block allocation, where a block of contiguous subcarriers are allocated to each user.

There has been extensive research in CFO compensation methods. The single-user detection (SUD) analyzed in [6] was the first carrier compensation technique that compensates for each user's CFO independently prior to applying a discrete Fourier transform (DFT) demodulator. Since in the SUD, each user requires a distinct DFT demodulator, the BS receiver complexity increases linearly with the number

Manuscript received October 27, 2010; accepted December 24, 2010. Date of publication January 06, 2011; date of current version March 09, 2011. The associate editor coordinating the review of this manuscript and approving it for publication was Prof. Ye (Geoffrey) Li. This work has been partially supported by the National Science Foundation under the award number 0801641.

H. Saeedi-Sourck and S. Sadri are with the Electrical and Computer Engineering Department, Isfahan University of Technology, Isfahan 84156, Iran (e-mail: saeedi@ec.iut.ac.ir; sadri@cc.iut.ac.ir).

Y. Wu and J. W. M. Bergmans are with the EE Department, Eindhoven University of Technology, Eindhoven $5600 \mathrm{MB}$, The Netherlands (e-mail: y.w.wu@tue.nl; j.w.m.bergmans@tue.nl).

B. Farhang-Boroujeny is with the Electrical Engineering Department, University of Utah, Salt Lake City, UT 84112 USA (e-mail: farhang@ece.utah.edu).

Digital Object Identifier 10.1109/TSP.2010.2104148 of users. Choi et al. proposed a CFO compensation method after DFT, noting that multiplication of two vectors before DFT is equivalent to the circular convolution of the associated vectors after DFT [7]. This method is usually referred to as CLJL, which stands for the initials of the authors-Choi-Lee-Jung-Lee.

Both SUD and CLJL techniques compensate for each user's CFO without considering any MAI cancellation [3]. Some parallel interference cancellation (PIC) techniques have been proposed to reduce MAI after SUD and CLJL processing, e.g., [8]-[11]. Huang and Letaief [8] proposed the addition of a PIC to the CLJL method. Similarly, a PIC may be adopted to reduce MAI after DFT block in SUD [9]. Successive interference cancellation (SIC) and linear multiuser detectors have also been suggested in [10] and [11], respectively. These result in some performance gain, however, at a cost of significant increase in complexity [3], [10]. In the rest of this paper, we use the names pre-DFT to refer to the SUD method of [6] and post-DFT to refer to the CLJL method of [7]. Also, the suffix PIC is added to these when a parallel interference cancellation is added.

None of the above techniques can correct the CFO for the uplink of an OFDMA perfectly. The interference cancellation (IC) techniques mitigate MAI to an extent, but not perfectly [3]. They also add significant computational complexity to the BS receiver. This paper studies an alternative method that resolves the problem of MAI in a more natural way. We propose using filter bank multicarrier (FBMC) modulation as an alternative technique to OFDM. The advantages of FBMC over OFDM in cognitive radios have recently been discussed in [12]. In this paper, we emphasize on the advantages of FBMC over OFDM in the uplink of a multiuser network. We note that the low side-lobe filters used in FBMC in a natural way leads to an effective MAI cancellation at virtually no additional cost-no IC is needed. At the same time, we note that although in a point-to-point (i.e., single-user) communication system FBMC is more complex than OFDM, in a multiuser system, the added complexity arising from IC blocks in an OFDMA system makes it significantly more complex than its FBMC counterpart.

There are a few choices for FBMC. The emphasis of this paper is on a type of FBMC that has been widely studied in the literature and often referred to as OFDM/OQAM where OQAM stands for offset quadrature amplitude modulation [13]. The word offset reflects the fact that, in OQAM, the in-phase and quadrature components of each data symbol have a time-offset of half a symbol interval. In [13] it has been noted that the term staggered QAM has also been used to refer to OQAM and accordingly suggests the more concise name staggered modulated multitone (SMT). Throughout this paper, we consider SMT for implementation of FBMC systems, and accordingly use the name SMTMA (SMT multiple access) when reference is made to FBMC-based multiple access networks.

Fusco et al. [14] have also noted the significance of SMTMA in the uplink of multiuser networks. A sensitivity analysis of SMTMA when different users are subject to different CFOs was presented in [14]. The emphasis of this paper, on the other hand, is on the complexity comparison of SMTMA and OFDMA in the uplink of a network. Here both pre-DFT and post-DFT CFO correction techniques are studied in detail. Our study of post-DFT CFO correction leads to a reduced complexity implementation of the SMTMA system. Simulation results that show the performance comparison between SMTMA and OFDMA, with different CFO compensation techniques, confirm superior performance of the former.

This paper is organized as follows. The system model is presented in Section II. To facilitate the complexity comparison of SMTMA 


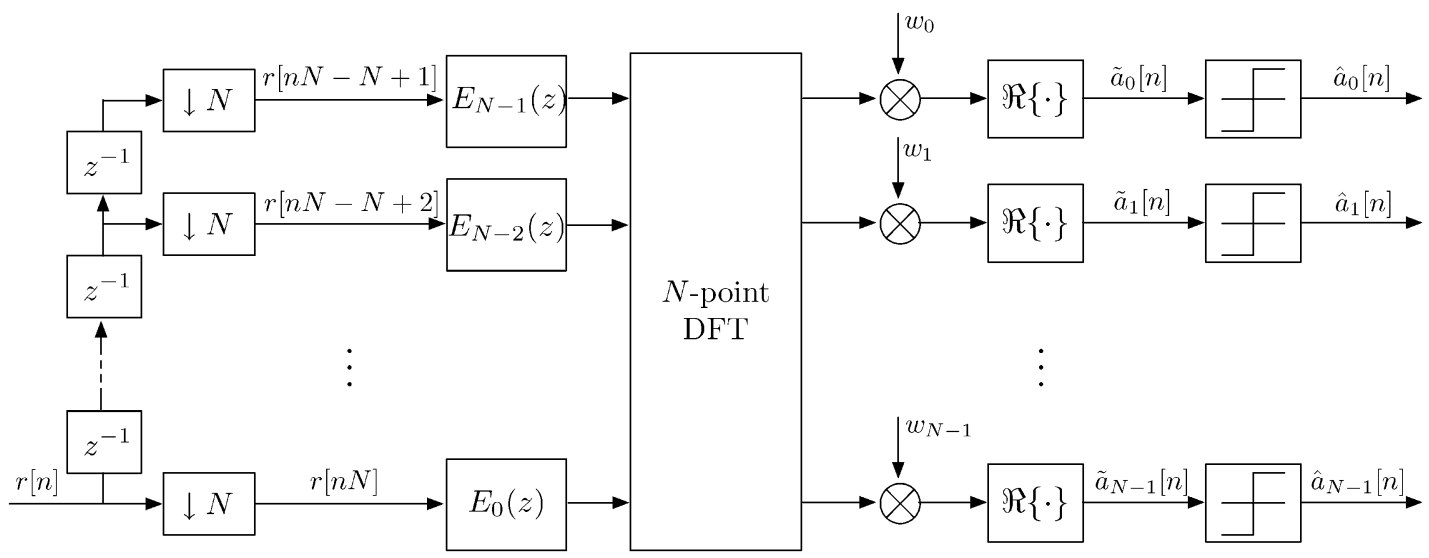

Fig. 1. The in-phase part of an SMT receiver. The quadrature part has a similar structure with the following minor differences: i) The input is backward delayed by one half of a symbol interval (N/2 samples), and ii) the $\Re\{\cdot\}$ blocks are replaced by $\Im\{\cdot\}$ blocks. $\Re\{\cdot\}$ denotes the real part of and $\Im\{\cdot\}$ means the imaginary part of.

and OFDMA, a polyphase structure of SMT receiver is reviewed in Section III. In Section IV, the CFO compensation methods that could be applied to SMTMA are developed. The computational complexity comparison of OFDMA and SMTMA methods are presented in Section V. Simulation results are presented in Section VI and the conclusions of the paper are drawn in Section VII.

\section{SYSTEM SETUP}

We consider the uplink of a multicarrier multiuser network where $P$ active users are communicating with a BS. This network may be based on OFDMA or SMTMA. We assume that there are $N=P \cdot Q$ subcarriers, where $Q$ is the number of subcarriers allocated to each user, including both active and null subcarriers. The set of $Q$ subcarriers assigned to the $p$ th user is denoted by $S_{p}$, and we assume $\bigcup_{p=0}^{P-1} S_{p}=$ $\{0,1, \cdots, N-1\}$ and $S_{p} \cap S_{q}=\varnothing$, for $\forall p \neq q$. The transmitted signal from the $p$ th user is represented by $x_{p}[n]$, and the channel response between the $p$ th user and the $\mathrm{BS}$ is denoted by the sequence $c_{p}[n]$. It is assumed that $c_{p}[n]$ is nonzero only for $n=0,1, \cdots, D-1$, where $D$ is the maximum channel delay spread. Accordingly, the received signal can be written as

$$
r[n]=\sum_{p=0}^{P-1}\left(x_{p}[n] \star c_{p}[n]\right) e^{j 2 \pi \varepsilon_{p} n / N}+\nu[n]
$$

where $\star$ denotes linear convolution, $\varepsilon_{p}, p=0, \cdots, P-1$ is the normalized CFO (with respect to the carrier spacing) corresponding to the $p$ th user, and $\nu[n]$ is an additive white Gaussian noise.

\section{Polyphase StRUcture OF SMT RecEIVER}

Several polyphase structures have been proposed for the implementations of SMT systems, e.g., [15]-[17]. The structures proposed in [15] and [16] have the same computational complexity. On the other hand, [17] proposes a structure with half computational complexity of those in [15] and [16]. However, unfortunately, careful examination of the structure in [17] reveals that certain symmetry properties used to simplify the implementation do not hold in the presence a generic channel. This renders the proposed structure unusable in practical implementation of SMT receivers. For the purpose of our study, in this paper we have chosen the polyphase structure of [15].

Fig. 1 presents the in-phase part of the polyphase structure proposed in [15]. This structure assumes that there is no CFO and thus it con- tains no CFO compensation block. $E_{0}(z)$ through $E_{N-1}(z)$ are the polyphase components of a prototype filter $H(z)$ based on which the SMT is implemented. We assume that $H(z)$ has a length of $L \cdot N$, thus, each polyphase component has a length of $L$. The DFT block takes care of the demodulation part of the system. Although in SMT, in general, the equalizer at each subcarrier may be a multi-tap transversal filter [18], in this work single-tap equalizers (the multipliers $w_{0}$ through $\left.w_{N-1}\right)$ are used. This is a reasonable assumption, as in practice one may always increase $N$ so that each subcarrier channel can be well approximated by a flat gain [19].

It is apparent that Fig. 1 is different from a conventional analysis filter bank as appears in the literature where the polyphase components are often followed by an IDFT, [15], [20]. This difference arises, simply, because here we have chosen to feed the input signal from the bottom of a tapped delay line, i.e., opposite to the common practice where the tapped delay line is fed from the top. This puts the input samples in a reversed order and accordingly the IDFT has to be replaced by DFT. This arrangement is chosen, here, because it matches the common practice in presentation of OFDM receiver.

The $N \times 1$ input vector to the DFT block at the time instant $n$ may be written as

$$
\mathbf{y}[n]=\sum_{i=1}^{L} \mathbf{y}^{(i)}[n]
$$

where

$$
\begin{aligned}
& \mathbf{y}^{(i)}[n]=\mathbf{r}[n+i] \odot \mathbf{h}[L-i], \\
& \mathbf{h}[l]=\left[\begin{array}{c}
h[l N+N-1] \\
h[l N+N-2] \\
\vdots \\
h[l N]
\end{array}\right], \mathbf{r}[n]=\left[\begin{array}{c}
r[n N-N+1] \\
r[n N-N+2] \\
\vdots \\
r[n N]
\end{array}\right],
\end{aligned}
$$

and $\odot$ denotes point-wise multiplication of vectors.

Using (2) and following the block diagram of Fig. 1, one finds that the input vector to the slicers is given by

$$
\tilde{\mathbf{a}}[n]=\Re\{\mathbf{w} \odot \mathcal{F}(\mathbf{y}[n])\}=\Re\left\{\mathbf{w} \odot \mathcal{F}\left(\sum_{i=1}^{L} \mathbf{y}^{(i)}[n]\right)\right\}
$$


where $\mathbf{w}=\left[\begin{array}{llll}w_{0} & w_{1} & \cdots & w_{N-1}\end{array}\right]^{T}$ is the column vector of the equalizer coefficients, $\mathcal{F}$ denotes the DFT operation, and $\mathbf{a}[n]$ is the vector of the recovered data symbols $\tilde{a}_{k}[n], k=0, \cdots, N-1$, before being passed to the slicers whose outputs are the final decisions $\hat{a}_{k}[n]$ of the transmitted data symbols. In a multiple access system, each set of subcarriers belongs to one user, hence, each set of elements of a $[n]$ belongs to a different user.

\section{CFO COMPENSATION METHODS FOR SMTMA}

We assume that the receiver (the BS) has perfect knowledge of CFOs of all users. As in SMT each subcarrier may receive MAI only from the nearby bands, thanks to the very low side lobes of its prototype filter, no IC is required. MAI is avoided, simply, by adding a few null subcarriers at the edges of each set of subcarriers that have been allocated to each user.

\section{A. Pre-DFT Compensation}

This is equivalent to the SUD method in OFDMA. The CFO of each user is compensated before applying the analysis filter bank/the demodulator. For the $p$ th user, the CFO compensated signal is formed as

$$
y_{p}[n]=r[n] e^{-j 2 \pi \hat{\varepsilon}_{p} n / N}
$$

where $\hat{\varepsilon}_{p}$ is an estimate of the CFO of the $p$ th user. The CFO compensated signal $y_{p}[n]$ is then analyzed to extract the data transmitted from the $p$ th user. In other words, detection of the data from each user requires a separate polyphase filter bank. Implementation of this polyphase structure is mathematically summarized as

$$
\tilde{\mathbf{a}}_{p}[n]=\Re\left\{e^{-j 2 \pi \hat{\varepsilon}_{p} n} \mathbf{w} \odot \mathcal{F}\left(\sum_{i=1}^{L} e^{-j 2 \pi \hat{\varepsilon}_{p} i} \mathbf{c}\left(\hat{\varepsilon}_{p}\right) \odot \mathbf{y}^{(i)}[n]\right)\right\}
$$

where

$$
\mathbf{c}\left(\hat{\varepsilon}_{p}\right)=\left[\begin{array}{c}
e^{j 2 \pi \hat{\varepsilon}_{p}(N-1) / N} \\
e^{j 2 \pi \hat{\varepsilon}_{p}(N-2) / N} \\
\vdots \\
1
\end{array}\right],
$$

and the subscript $p$ has been added to $\tilde{\mathbf{a}}[n]$ to indicate that it contains the recovered data of the $p$ th user. Clearly, one is only interested in the elements of $\tilde{\mathbf{a}}_{p}[n]$ that correspond to the subcarrier outputs associated with the present user.

\section{B. Post-DFT Compensation}

In the pre-DFT CFO compensation, detection of the data from each user requires a separate analysis filter bank. This increases the computational complexity as the number of users increases. To resolve this problem, as proposed in [7], for OFDMA, one may compensate CFO after DFT. We follow the same philosophy here and develop a post-DFT CFO compensation method for SMTMA.

Straightforward manipulations of (5) lead to

$$
\begin{array}{r}
\tilde{\mathbf{a}}_{p}[n]=\Re\left\{e ^ { - j 2 \pi \hat { \varepsilon } _ { p } n } \mathbf { w } \odot \left[\left(\sum_{i=1}^{L} e^{-j 2 \pi \hat{\varepsilon}_{p} i} \mathcal{F}\left(\mathbf{y}^{(i)}[n]\right)\right)\right.\right. \\
\left.\left.\circledast \mathcal{F}\left(\mathbf{c}\left(\hat{\varepsilon}_{p}\right)\right)\right]\right\}
\end{array}
$$

where $\circledast$ denotes circular convolution. This result shows how one may apply CFO compensation for each user, after converting each column vector $\mathbf{y}^{(i)}[n]$ to the frequency domain. One may notice that while (5) involves one DFT operation per user, (7) has to perform $L$ DFT operations that can be shared among all users. However, we need to remove the phase component $2 \pi \hat{\varepsilon}_{p} i$ for each user separately. This adds some complexity to the receiver which increases with the number of users. Additional complexity is required to obtain $\mathcal{F}\left(\mathbf{c}\left(\hat{\varepsilon}_{p}\right)\right)$. To resolve this problem, in the following, we develop an alternative structure.

We refer to (5) and note that the vector

$$
\mathbf{z}[n]=\sum_{i=1}^{L} e^{-j 2 \pi \hat{\varepsilon} p^{i}} \mathbf{c}\left(\hat{\varepsilon}_{p}\right) \odot \mathbf{y}^{(i)}[n]
$$

can be obtained by defining the $L$ times taller vectors

$$
\begin{aligned}
& \mathbf{c}^{\prime}\left(\hat{\varepsilon}_{p}\right)= {\left[\begin{array}{c}
e^{-j 2 \pi \hat{\varepsilon}_{p}} \mathbf{c}\left(\hat{\varepsilon}_{p}\right) \\
e^{-j 4 \pi \hat{\varepsilon}_{p}} \mathbf{c}\left(\hat{\varepsilon}_{p}\right) \\
\vdots \\
e^{-j 2 \pi \hat{\varepsilon}_{p} L} \mathbf{c}\left(\hat{\varepsilon}_{p}\right)
\end{array}\right]=\left[\begin{array}{c}
e^{-j 2 \pi \hat{\varepsilon}_{p} / N} \\
e^{-j 4 \pi \hat{\varepsilon}_{p} / N} \\
\vdots \\
e^{-j 2 \pi \hat{\varepsilon}_{p} L N / N}
\end{array}\right], } \\
& \mathbf{y}^{\prime}[n]=\left[\begin{array}{c}
\mathbf{y}^{(1)}[n] \\
\mathbf{y}^{(2)}[n] \\
\vdots \\
\mathbf{y}^{(L)}[n]
\end{array}\right],
\end{aligned}
$$

and $\mathbf{z}^{\prime}[n]=\mathbf{c}^{\prime}\left(\hat{\varepsilon}_{p}\right) \odot \mathbf{y}^{\prime}[n]$, and then adding the successive length $N$ partitions of $\mathbf{z}^{\prime}[n]$. This last step may be viewed as an aliasing in the time domain. Its equivalent in the frequency domain is to take the $L$-fold decimated samples of the DFT of $\mathbf{z}^{\prime}[n]$.

From the above result, we conclude that (5) can be rearranged as

$$
\tilde{\mathbf{a}}_{p}[n]=\Re\left\{e^{-j 2 \pi \hat{\varepsilon}_{p} n} \mathbf{w} \odot\left[\mathcal{F}\left(\mathbf{c}^{\prime}\left(\hat{\varepsilon}_{p}\right) \odot \mathbf{y}^{\prime}[n]\right)\right]_{\downarrow L}\right\}
$$

where the subscript $\downarrow L$ means $L$-fold decimation. Noting that

$$
\mathcal{F}\left(\mathbf{c}^{\prime}\left(\hat{\varepsilon}_{p}\right) \odot \mathbf{y}^{\prime}[n]\right)=\mathcal{F}\left(\mathbf{y}^{\prime}[n]\right) \circledast \mathcal{F}\left(\mathbf{c}^{\prime}\left(\hat{\varepsilon}_{p}\right)\right),
$$

(9) can be written as

$$
\tilde{\mathbf{a}}_{p}[n]=\Re\left\{e^{-j 2 \pi \hat{\varepsilon}_{p} n} \mathbf{w} \odot\left[\mathcal{F}\left(\mathbf{y}^{\prime}[n]\right) \circledast \mathcal{F}\left(\mathbf{c}^{\prime}\left(\hat{\varepsilon}_{p}\right)\right)\right]_{\downarrow L}\right\} .
$$

\section{Computational COMPleXity}

Table I summarizes the computational complexity of OFDMA, as presented in [7] and [9], and also that of SMTMA for different CFO compensation techniques. It is assumed that there are $P$ users and $N / P$ subcarriers are assigned to each user from a total of $N$. All operations involve complex numbers and the complexity expressions refer to the number of complex multiplications (CMs) for each case. Also, to give an idea of what these expressions lead to in practical systems, the respective computational complexities are calculated and listed in Table II, for two typical cases of $N=256$ and 2048, following the IEEE 802.16e specifications [21]. As seen, while OFDMA without PIC 
TABLE I

COMPUTATIONAL COMPLEXITY OF SMTMA AND OFDMA WiTH THE CFO COMPENSATION TECHNIQUES. $N$ : NumBER OF SUBCARRIERS, $P$ : NuMBER OF USERS, $m$ : Stage IndeX/Number of Cancellation Iterations, $N L$ : Prototype Filter Length, $M$ : Number of Non Zero Elements in $\mathbf{C}^{\prime}\left(\hat{\varepsilon}_{p}\right)$

\begin{tabular}{|c|c|}
\hline Compensator & Computational complexity \\
\hline \hline pre-DFT OFDMA, [7] & $\frac{N P}{2} \log _{2} N-\left\{\frac{N P}{2} \log _{2} P-\frac{3}{2} N(P-1)\right\}$ \\
\hline post-DFT OFDMA, [7] & $\frac{N}{2} \log _{2} N+\frac{N^{2}}{P}$ \\
\hline pre-DFT OFDMA with PIC, [9] & $\frac{N P}{2} \log _{2} N-\left\{\frac{N P}{2} \log _{2} P-\frac{3}{2} N(P-1)\right\}+$ \\
& $(m-1)\left(N^{2}-\frac{N^{2}}{P}\right)$ \\
\hline post-DFT OFDMA with PIC, [9] & $\frac{N}{2} \log _{2} N+\frac{N^{2}}{P}+(m-1)\left(N^{2}+\frac{N^{2}}{P}\right)$ \\
\hline pre-DFT SMTMA & $N L P+N P \log _{2} N-\left(N P \log _{2} P-2 N(P-1)\right)$ \\
\hline post-DFT SMTMA & $N L+N L \log _{2} N L+2 N M$ \\
\hline
\end{tabular}

TABLE II

COMPUTATIONAL COMPLEXITY of SMTMA AND OFDMA WITH THE CFO COMPENSATION TECHNIQUES. $m=3, L=3, M=20$

\begin{tabular}{|c|ccc|ccc|}
\hline Type & \multicolumn{3}{|c|}{$N=256$} & \multicolumn{3}{c|}{$N=2048$} \\
\hline & $P=2$ & $P=4$ & $P=16$ & $P=2$ & $P=4$ & $P=16$ \\
\hline \hline pre-DFT OFDMA & 2,176 & 4,224 & 13,956 & 23,552 & 46,080 & 160,768 \\
\hline post-DFT OFDMA & 33,792 & 17,408 & 5,120 & $2,108,416$ & $1,059,840$ & 273,408 \\
\hline pre-DFT OFDMA with PIC & 67,712 & 102,528 & 136,832 & $4,217,856$ & $6,337,536$ & $8,025,088$ \\
\hline post-DFT OFDMA with PIC & 230,400 & 181,248 & 144,384 & $14,691,328$ & $11,545,600$ & $9,186,304$ \\
\hline pre-DFT SMTMA & 5,632 & 10,752 & 36,352 & 57,344 & 110,592 & 389,120 \\
\hline post-DFT SMTMA & 18,369 & 18,369 & 18,369 & 165,390 & 165,390 & 165,390 \\
\hline \hline
\end{tabular}

offers a lower complexity than SMTMA, the addition of PIC to the former leads to a significantly higher complexity.

The computational complexity expressions for SMTMA are obtained as follows. In the case of pre-DFT compensation, $P$ DFTs, each of size $N$, have to be performed, for each of the phase and quadrature parts of the receiver. Assuming that $N$ is a power of 2 and FFT technique is used, this leads to $2 \times P \times(N / 2) \log _{2} N=N P \log _{2} N$ CMs. NP CMs are needed for the CFO compensation. Other terms are minor adjustment to the number of operations whose details are left to the interested readers.

In the case of post-DFT compensation, the major operations are two DFTs, each of size $N L$, which has to be performed to recover a block of $N$ QAM data symbols. This requires $N L \log _{2} N L$ CMs. The implementation of each circular convolution will lead to the complexity number $N^{2} L^{2}$. This should be repeated $2 P$ times (for phase and quadrature parts of each of $P$ users) and leads to a total of $2 P N^{2} L^{2}$ CMs. This surpasses the complexity of all the other systems listed in Table I. However, a closer look at the actual output samples which are necessary for each user reveal that this reduces to $2 P N L \times(N / P)=2 N^{2} L$ CMs. Further computational saving is made by noting that for typical values of $N L$ (in the order of 1000 or larger), a large percentage of the elements of $\mathcal{F}\left(\mathbf{c}^{\prime}\left(\hat{\varepsilon}_{p}\right)\right)$ are very close to zero, and thus can be substituted by zero, [7], [8]. This special structure of $\boldsymbol{F}\left(\mathbf{c}^{\prime}\left(\hat{\varepsilon}_{p}\right)\right)$ comes from the fact that $\mathbf{c}^{\prime}\left(\hat{\varepsilon}_{p}\right)$ is a complex sine-wave with the frequency $\hat{\varepsilon}_{p}$, thus, the non-zero samples of its Fourier transform are concentrated around zero. Numerical evaluations reveal that, for the cases of interest (such as those in Table II), the number of significant elements of $\mathcal{F}\left(\mathbf{c}^{\prime}\left(\hat{\varepsilon}_{p}\right)\right)$, that we denote by $M$, is approximately independent of $N$. For instance, the choice of $M=20$, for both cases of $N=256$ and 2048, assures that for any value of $\hat{\varepsilon}_{p}$ in the range of -0.5 to $+0.5,98 \%$ of the energy of samples of $\mathcal{F}\left(\mathbf{c}^{\prime}\left(\hat{\varepsilon}_{p}\right)\right)$ remain within its first $M / 2$ and last $M / 2$ samples. This choice of $M$ in Table II has been on this basis and the fact that this value of $M$

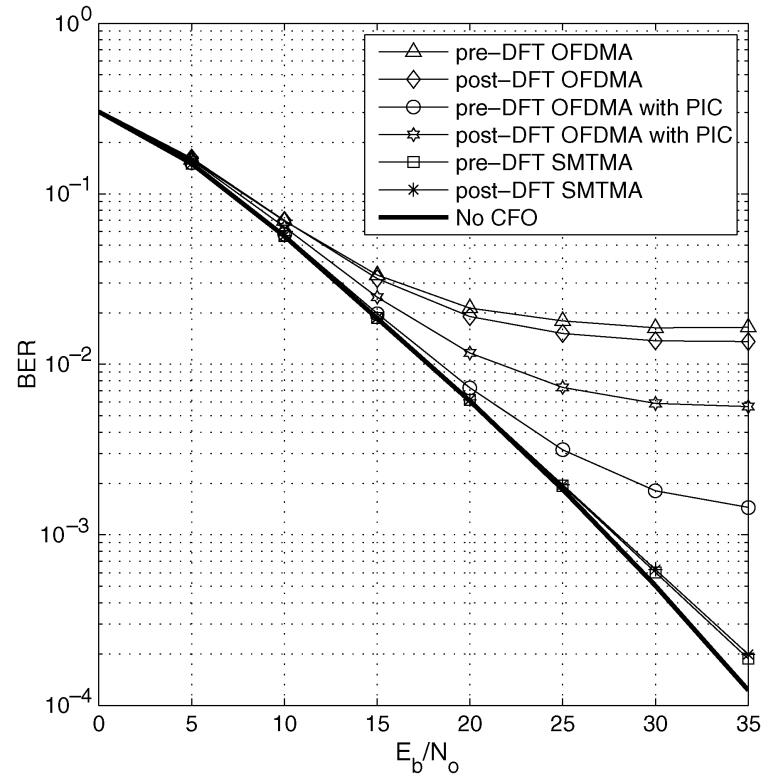

Fig. 2. Coded BER performance for SMTMA and OFDMA when all users signals reach the BS with the same power.

incurs no noticeable loss in the BER curves of post-DFT SMTMA; see Fig. 2, below. Excluding the near zero elements of $\mathcal{F}\left(\mathbf{c}^{\prime}\left(\hat{\varepsilon}_{p}\right)\right)$ in performing the circular convolutions, further reduces the number of CMs from $2 N^{2} L$ to $2 M N$.

The numerical values presented in Table II show that pre- and post-DFT OFDMA systems without PIC have the lowest complexity. However, as demonstrated in the next section, these methods perform very poorly. Addition of PIC to these systems increase the complexity by an order of magnitude, when $N=256$, and two orders of 


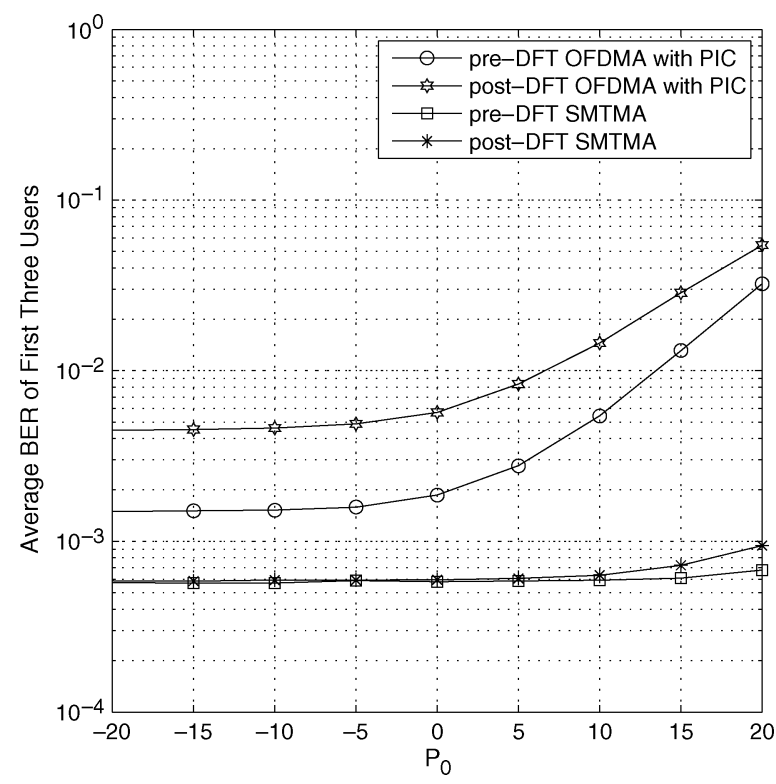

Fig. 3. Coded BER performance for SMTMA and OFDMA when signals from three of four users reach the BS with the same power and the power of the forth user deviates by $P_{0} \mathrm{~dB}$.

magnitude, when $N=2048$. The complexity of pre- and post-DFT SMTMA systems lies between those of OFDMA with and without PIC, and usually closer to the latter. Pre-DFT SMTMA has a lower complexity than its post-DFT counterpart, for smaller numbers of users, $P$. However, the situation reverses when $P>6$.

\section{Simulation Results}

Computer simulations are performed to compare the performance of SMTMA and OFDMA. We let $N=256$ and assume that there are $P=4$ users in the network, hence, $Q=N / P=64$ subcarriers per user. The multipath channel SUI-2 proposed by the IEEE802.16 broadband wireless access working group [22] is considered. The $\mathrm{CFO}$ values are chosen randomly and independently for each user from a uniform distribution in the interval $-0.5<\varepsilon<0.5$. In all cases, one guard subcarrier is inserted between each pair of adjacent users's bands. Perfect power control is assumed. SMTMA systems use a SR-Nyquist prototype filter with a length of $3 N$, designed following [23]. The channel code is a rate $1 / 2$ convolutional code with a constraint length of 5 and data symbols are from a 16-QAM constellation. The bit error rate (BER) results are presented in Fig. 2, where $E_{b} / N_{0}$ denotes bit power over noise power. As seen, OFDMA without PIC performs poorly. An error floor of around $10^{-2}$ is observed. PIC helps to reduce the error floor, but the performance still remains relatively poor. SMTMA systems, on the other hand, remain solid and follow the reference curve, obtained when all users are perfectly carrier synchronized.

To demonstrate the effect of a possible imperfect power control situation, we run the following experiment. We assume that from the four users, three have perfect power control, thus their respective signals reach the BS with the same power. However, the forth user's power differs from the others by $P_{0} \mathrm{~dB}$. Fig. 3 presents the average BERs of the first three users for the case where for them $E_{b} / N_{0}=30 \mathrm{~dB}$, and $P_{0}$ varies from -20 to $+20 \mathrm{~dB}$. As seen, in this case, while SMTMA performance remains almost unaffected by variation of $P_{0}$, OFDMA degradation is significant.

\section{CONCLUSION}

A study of FBMC for implementation of the uplink of a multicarrier multiuser network was presented. We noted that although OFDMA has been proposed as a candidate in the majority of present standards, an FBMC-based implementation leads to a much superior performance and a lower computational complexity. This is a consequence of the fact that FBMC uses near perfect filters to separate subcarriers/users, thus, avoids the need for any interference cancellation.

\section{REFERENCES}

[1] A. Larmo, M. Lindstrom, M. Meyer, G. Pelletier, J. Torsner, and H. Wiemann, "The LTE link-layer design," IEEE Commun. Mag., vol. 47, no. 4, pp. 52-59, Apr. 2009.

[2] A. M. Tonello, N. Laurenti, and S. Pupolin, "Analysis of the uplink of an asynchronous multiuser DMT OFDMA system impaired by time offsets, frequency offsets, and multipath fading," in Proc. Veh. Technol. Conf., Oct. 2000, vol. 3, pp. 1094-1099.

[3] B. M. Morelli, C.C. J. Kuo, and M. N. Pun, "Synchronization techniques for orthogonal frequency division multiple access (OFDMA): A tutorial review," Proc. IEEE, vol. 95, no. 7, pp. 1394-1427, Jul. 2007.

[4] G. Berardinelli, L. A. Ruiz de Temino, S. Frattasi, M. Rahman, and P. Mogensen, "OFDMA versus SC-FDMA: Performance comparison in local area IMT-A scenarios," IEEE Wireless Commun., vol. 15, no. 5, pp. 64-72, Oct. 2008.

[5] M. Rinne, M. Kuusela, E. Tuomaala, P. Kinnunen, I. Kovacs, K. Pajukoski, and J. Ojala, "A performance summary of the evolved $3 \mathrm{G}$ (E-UTRA) for voice over internet and best effort traffic," IEEE Trans. Veh. Technol., vol. 58, no. 7, pp. 3661-3673, Sep. 2009.

[6] A. Tonello and S. Pupolin, "Performance of single user detectors in multitone multiple access asynchronous communications," in Proc. IEEE Veh. Technol. Conf., May 2002, pp. 199-203.

[7] J. Choi, C. Lee, H. W. Jung, and Y. H. Lee, "Carrier frequency offset compensation for uplink of OFDM-FDMA systems," IEEE Commun. Lett., vol. 4, no. 12, pp. 414-416, Dec. 2000.

[8] D. Huang and K. B. Letaief, "An interference cancellation scheme for carrier frequency offsets correction in OFDMA systems," IEEE Trans. Commun., vol. 53, no. 7, pp. 1155-1165, Jul. 2005.

[9] S. Manohar, D. Dheeraj, V. Tikiya, and A. Chokalingam, "Cancellation of multiuser interference due to carrier frequency offsets in uplink OFDMA," IEEE Trans. Wireless Commun., vol. 6, no. 7, pp. 2560-2571, Jul. 2007.

[10] T. Yücek and H. Arslan, "Carrier frequency offset compensation with successive cancellation in uplink OFDMA systems," IEEE Trans. Wireless Commun., vol. 6, no. 10, pp. 3546-3551, Oct. 2007.

[11] Z. Cao, U. Tureli, Y. D. Yao, and P. Honan, "Low-Complexity orthogonal spectral signal construction for generalized OFDMA uplink with frequency synchronization errors," IEEE Trans. Veh. Technol., vol. 56, no. 3, pp. 1143-1154, May 2007.

[12] B. Farhang-Boroujeny and R. Kempter, "Multicarrier communication techniques for spectrum sensing and communication in cognitive radios," IEEE Commun. Mag. (Special Issue on Cognitive Radios for Dynamic Spectrum Access), vol. 46, no. 4, pp. 80-85, Apr. 2008.

[13] B. Farhang-Boroujeny and C. H. Yuen, "Cosine modulated and offset QAM filter bank multicarrier techniques: A continuous-time prospect," EURASIP J Appl Signal Process. (Special Issue on Filter Banks for Next Generation Multicarrier Wireless Communications), 2010, 10.1155/2010/165654, Article ID 165654, 16 pp.

[14] T. Fusco, A. Petrella, and M. Tanda, "Sensitivity of multi-user filterbank multicarrier systems to synchronization errors," in Proc. IEEE ISCCSP, Mar. 2008, pp. 393-398.

[15] N. J. Fleige, Multirate Digital Signal Processing. New York: Wiley, 1994.

[16] P. Siohan, C. Siclet, and N. Lacaille, "Analysis and design of OFDM/OQAM systems based on filterbank theory," IEEE Trans. Signal Process., vol. 50, no. 5, pp. 1170-1183, May 2002. 
[17] L. Vangelista and N. Laurenti, "Efficient implementations and alternative architectures for OFDM-OQAM systems," IEEE Trans. Commun., vol. 49, no. 4, pp. 664-675, Apr. 2001.

[18] B. Hirosaki, "An orthogonally multiplexed QAM system using the discrete Fourier transform," IEEE Trans. Commun., vol. C-29, no. 7, pp. 982-989, Jul. 1981.

[19] B. Farhang-Boroujeny, "Multicarrier modulation with blind detection capability using cosine modulated filter banks," IEEE Trans. Commun., vol. 51, no. 12, pp. 2057-2070, Dec. 2003.

[20] P. P. Vaidyanathan, Multirate Systems and Filter Banks. Englewood Cliffs, NJ: Prentice-Hall, 1993.
[21] Part 16: Air Interface For Fixed and Mobile Broadband Wireless Access Syst. Amendment 2: Phys. and Medium Access Control Layers For Combined Fixed and Mobile Oper. in Licensed Bands and Corrigendum 1, IEEE 802.16e-2005, Feb. 2006.

[22] The IEEE802.16 Broadband Wireless Access Working Group, Channel Models for Fixed Wireless Applications [Online]. Available: www. ieee802.org/16/tg3/contrib/802163c-0129r4.pdf

[23] B. Farhang-Boroujeny, "A square-root nyquist (M) filter design for digital communication systems," IEEE Trans. Signal Process., vol. 56, no. 5, pp. 2127-2132, May 2008. 